\section{miR-22-3p Suppresses Adipocyte Formation of Telomerase Transformed Mesenchymal Stromal Cells (iMSC3) by Regulating Non- targeted MAPK Signaling Pathway}

\author{
Sara Sultan Alomran', Muhammad Nasir Khan Khattak ${ }^{1,2}$, Amir Ali Khan ${ }^{1,2 *}$, \\ Sallam Hasan Abdallah ${ }^{2}$, Abeer Maher Fayyad ${ }^{1}$ and Khalid Bajou ${ }^{1,2}$ \\ ${ }^{1}$ Department of Applied Biology, University of Sharjah, Sharjah, United Arab Emirates \\ ${ }^{2}$ Human Genetics and Stem Cells Research Group, Research Institute of Sciences and \\ Engineering, University of Sharjah, Sharjah, United Arab Emirates
}

\begin{abstract}
A B S T R A C T
Unraveling molecular mechanisms that govern adipocyte formation will lead to a greater understanding of obesity and subsequent treatment. In the current study, we assessed the effects of miR-22-3p, a non-coding RNA, on adipocyte differentiation from telomerase-transformed Mesenchymal Stromal Cells (iMSC3). The transfection of iMSC3 with miR-22-3p suppressed adipocyte differentiation as evident by reduction in lipid content and number of lipid droplets. While AKT3 is reported to be a target of miR-22-3p, our study indicated otherwise. Moreover, while miR-22-3p is known to target more than 500 genes, MAPK has not been identified as one of the more than 500 gene targets of miR-22-3p, though this pathway was upregulated in transfected iMSC3 that differentiated to adipocytes. Finally, miR-22-3p was observed to suppress adipocytes and was involved in adipocyte differentiation through non-targeted MAPK pathway upregulation rather than AKT3-related processes.
\end{abstract}

Article Information
Received 27 November 2019
Revised 04 February 2020
Accepted 20 February 2020
Available online 04 June 2021
Authors' Contribution
SSA and AAK conceived the project.
SSA, MNKK, SHA, AMF, KB and
AAK executed the experimental work
and analyzed the data. SSA, MNKK,
KB and AAK wrote the article. AAK
secured the funding and supervised
the work.
Key words
miR-22-3p, iMSC3, Adipocytes,
MAPK, AKT3

\section{INTRODUCTION}

$\mathrm{O}^{\prime}$ besity is a chronic disorder with increasing worldwide prevalence due to lack of physical activity and imbalanced diets. It leads to other diseases including type 2 diabetes mellitus, insulin resistance and cancer (Noriyoki et al., 2011; Garg et al., 2014; Yang et al., 2016). It is characterized by increase in fat mass either by enhanced adipocyte size or number or a combination of both (Wang et al., 2014). There are two types of adipose tissue, white adipose tissues (WAT) and brown adipose tissues (BAT). WAT is responsible for energy storage (Noriyoki et al., 2011) while BAT is responsible for burning fat and generation of energy (Ruiz-Ojeda et al., 2016). Both types of adipose tissue are formed from the differentiation of mesenchymal stem cells (MSC); this differentiation is regulated by complex gene regulation and interaction of different signaling pathways. The differentiation process consists of MSC differentiation into lipoblasts, then pre-adipocytes and finally mature adipocytes (Gregoire et al., 1998).

In order to treat obesity and its related disorders, the

Corresponding author: amkhan@sharjah.ac.ae 0030-9923/2021/0004-1491 \$9.00/0

Copyright 2021 Zoological Society of Pakistan molecular mechanisms involved have to be elucidated. MicroRNA (miRNA) roles in obesity and obesity-related disorders have been of great interest in recent years (Ortega et al., 2013). miRNAs are small conserved non-coding RNA molecules that regulate mRNA by transcript degradation or inhibition of translation. Hence, they act as key regulators in diverse biological processes such as proliferation, apoptosis and cellular differentiation (Sousa-nunes and Somers, 2013). Furthermore, miRNAs regulate energy balance and homeostasis in adipose tissue by controlling several metabolic pathways (Hartig et al., 2015).

MicroRNA miR-22-3p, located on 17p13.3 in human chromosome, was found to play a role in the differentiation of mesenchymal stem cells into neural progenitor cells (Huat et al., 2015). This study found that introduction of insulin growth factor (IGF1) enhanced the quality of bone marrow MSCs (BMSCs) derived from neural progenitor cells (NPCs). Microarray analysis has also revealed that introduction of IGF1 enhanced survivability and cell proliferation of NPC by down regulating key miRNAs. Among these, miR-22-3p was also downregulated (Huat et al., 2015). Furthermore, miR-22-3p has been studied as a tumor-suppressor in many types of human cancers (Luo et al., 2017) and in cellular senescence in breast cancer cells (Xiong et al., 2010). 
Signaling pathways are involved in cell differentiation processes and early development. MAPK and PI3-K signaling pathways play essential roles in adipocyte differentiation and obesity, especially early stem cell proliferation stages such as differentiation and adipogenesis (Xiong et al., 2010; Han et al., 2017). Our previous study showed that rosiglitazone enhances adipogenesis, particularly brown adipogenesis via the activation of PI3-K and MAPK pathways during the differentiation of telomerase-transformed mesenchymal stem cells into adipocytes (Fayyad et al., 2019).

Aprevious study has shown that human adipose derived mesenchymal stem cells (hADMSC) transfected with miR-22 inhibited lipid droplet formation and suppressed the expression of adipogenic transcription factors and specific genes (Huang et al., 2012). However, literature is lacking on the effect of miR-22-3p on telomerasetransformed mesenchymal stromal cells (iMSC3) during adipogenesis. Moreover, the effects of miR-22-3p during iMSC 3 cell differentiation into adipocytes and regulation of the PI3-K and MAPK signaling pathways have not been elucidated yet. As mentioned earlier, our previous study reported that MAPK pathway activation is associated with enhanced adipogenesis (Fayyad et al., 2019). However, this pathway is not directly targeted by miR-22-3p but it is cross linked with the PI3-K pathway (Castellano and Downward, 2011; Aksamitiene et al., 2012). Hence, in this study, the inhibitory role of miR-22-3p in the in-vitro differentiation of iMSC 3 was investigated. We also studied the expression of MAPK and AKT proteins during the differentiation process in order to check if these pathways exhibited inhibitory or stimulatory roles.

\section{MATERIALS AND METHODS}

All methods were performed as described previously (Fayyad et al., 2019).

Maintenance of telomerase-transformed mesenchymal stromal (iMSC3) cell-line

Telomerase-transformed mesenchymal stromal cellline hTERT (iMSC3) (abm T0529, Canada) were grown in minimum essential medium eagle (MEM Eagle) (SigmaAldrich M2279, USA) supplemented with $10 \%$ fetal bovine serum (FBS), 1\% penicillin/ streptomycin (Pen/ Strep) and $200 \mu \mathrm{M}$ L-glutamine. Cells were incubated at $37^{\circ} \mathrm{C}$ in an incubator supplied with $5 \% \mathrm{CO}_{2}$.

\section{Transfection efficiency}

Using the RNAiMAX transfection kit (13778150, Thermo-fisher scientific, USA), iMSC3 cells were transfected with non-target miRNA sequences labeled with
CY3 (AM17120, Thermo-fisher scientific, USA). 10 $\mu \mathrm{M}$ of CY3-labeled microRNA was transfected to $250 \times 10^{3}$ cells seeded per well in a 6-well plate. Transfection efficiency was evaluated after $24 \mathrm{~h}$ using flow cytometry (BD FACS AREAIII, BD Bioscience, USA).

\section{Transfection and differentiation to adipocytes}

For iMSC3 differentiation into adipocytes in the presence of miR-22-3p mimic, $250 \times 10^{3}$ cells were seeded onto a 6-well plate. After 24-48 h, cells were transfected at $60-80 \%$ confluency (Fig. 1). iMSC3 cells were treated in three groups, group 1 was transfected with hsa-miR22-3p mimic (MC10203, Thermo-fisher scientific, USA) (miR-22-3p); group 2 was transfected with non-target miRNA sequence (4464058, Thermo-Fisher Scientific, USA) (negative control); group 3 was differentiated with no transfection as control. The cell cycle was synchronized on the day of transfection using media lacking Pen/Strep and FBS for cells (Zhenhui, 2012).

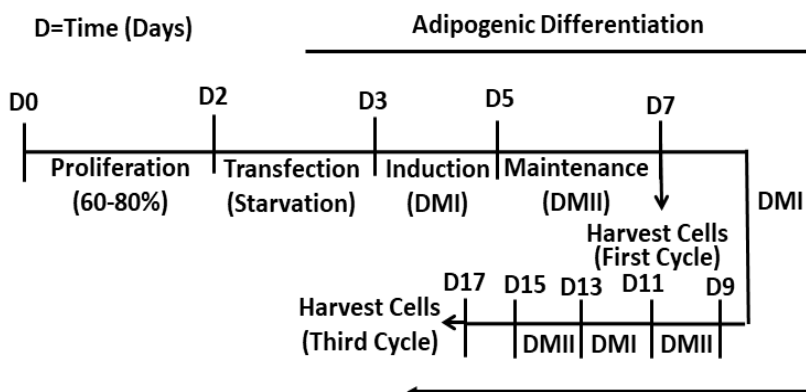

Fig. 1. Flow chart illustrating transfection and adipocyte differentiation of iMSC3 cells via methodology used in this study.

The differentiation cycle started $24 \mathrm{~h}$ following transfection and serum starvation. Three differentiation cycles were carried out and each differentiation cycle lasted for four days. iMSC3 cells were differentiated into adipocytes in induction medium (DMI) $[0.25 \mu \mathrm{M} /$ $\mathrm{mL}$ Dexamethasone (Sigma-Aldrich, USA), $0.1 \mu \mathrm{M} /$ $\mathrm{mL}$ Indomethacin (Sigma-Aldrich, USA), $0.5 \mu \mathrm{M} / \mathrm{m} \mathrm{L3-}$ isobutyl-1-methylxanthine (Sigma-Aldrich, USA), and 1 $\mu \mathrm{M} / \mathrm{mL}$ insulin] for $48 \mathrm{~h}$. Subsequently, cells were washed with PBS and maintained in maintenance medium (DMII) of $1 \mu \mathrm{M} / \mathrm{mL}$ insulin for $48 \mathrm{~h}$. The three differentiation cycles of induction and maintenance treatment lasted for 14 days. Cells were harvested for protein analysis at two time points: first cycle of differentiation (after 4 days) and at the third cycle (at the end of differentiation). The differentiation method utilized was similar to a previous study with some modifications (Zebisch et al., 2012). 


\section{Fixation of cells for staining}

Cells were washed twice with PBS and then incubated with $2 \mathrm{ml}$ cold $4 \%$ paraformaldehyde for 10 min. Paraformaldehyde was discarded and replaced with fresh $2 \mathrm{ml}$ cold 4\% paraformaldehyde, following which the cells were incubated for $1 \mathrm{~h}$ at room temperature.

\section{Nile red staining}

In Nile red staining, Nile red was used to stain fat droplets and DAPI to stain the nucleus of living cells. The staining method utilized was taken from a previous study with some modifications (Greenspan et al., 1985). Nile red stock solution was prepared in acetone $(1 \mathrm{mg} / \mathrm{ml})$, with the stain solution tightly sealed and protected from light at $4^{\circ} \mathrm{C}$. Working Nile red solution was prepared by diluting stock solution to $100 \mathrm{x}$ using trizma-maleate $(1 \mathrm{mM})$ (Sigma-Aldrich T3128, USA) and polyvinylpyrrolidone (3\% w/v) (Sigma-Aldrich P2307, USA). Fixed cells were stained with 1:100 Nile red stain and DAPI stain (Life Technologies P36930, Eugene, OR, USA). Fat droplet morphology in cells was observed under fluorescent microscope using Cellsens standard software.

\section{Oil red $O$ staining}

Oil red staining of fixed cells was performed to stain fat droplets in differentiated cells. The staining method was taken from a previous study with some modifications (Aguena et al., 2012). The derived adipocytes cells were washed twice with PBS, $2 \mathrm{ml}$ of cold $4 \%$ paraformaldehyde was added and the cells were incubated for $10 \mathrm{~min}$ at room temperature. Later, the solution was replaced with fresh $2 \mathrm{ml}$ of $4 \%$ cold paraformaldehyde and incubated for $1 \mathrm{~h}$ at room temperature. Following incubation, paraformaldehyde was removed, and cells were washed twice with distilled water. $2 \mathrm{ml}$ of $60 \%$ isopropanol was then added to cells for a further $10 \mathrm{~min}$ incubation period at room temperature. Cells were air-dried completely. Oil red O stain working solution (Sigma-Aldrich 1391, USA) was prepared by making $60 \%$ Oil red $\mathrm{O}$ stock solution and adding $2 \mathrm{ml}$ of filtered stain to cells followed by incubation for $2 \mathrm{~h}$ on shaker at room temperature. Following incubation, cells were washed three times with distilled water and $2 \mathrm{ml}$ of PBS was added for visualization under inverted light microscope using Optika vision lite software.

\section{Lipid content quantification}

Oil red O stain was eluted from cells using 100\% isopropanol and was incubated on a shaker at room temperature for $15 \mathrm{~min}$ (Aguena et al., 2012). 100 $\mu$ l of sample absorbance was quantified at 500nm (O.D) to detect the amount of stain attached to differentiated cell fat droplets in control and miR22-3p transfected cells, respectively.

\section{Fat droplet counting}

Utilizing visualized oil red $\mathrm{O}$ stained pictures showing clear lipid droplets, pictures were divided using gridlines and lipid droplets were quantified under the different treatment groups. Picture counting was performed thrice in 3 different pictures and the average percentage was calculated.

\section{Protein extraction and western blotting}

Total protein was extracted from pelleted differentiated cells by adding lysis buffer (which comprised of $50 \mathrm{mM}$ Tris $\mathrm{HCl} \mathrm{pH}=8.0,100 \mathrm{mM}$ sodium chloride $(\mathrm{NaCl}), 0.25 \%$ sodium dodecyl sulfate (SDS), $0.25 \%$ Triton X, 2mM ethylenediamine EDTA, $0.1 \%$ sodium deoxycholate and protease as well as phosphatase inhibitors), mixed well and incubated on ice. Samples were centrifuged to remove cellular debris. Protein amount was quantified via the DCTM Protein Assay (Bio-Rad 500-0116, Hercules, CA, USA) using the manufacturer's recommended protocol. Equal amount $(50 \mathrm{mg} / \mu \mathrm{l})$ of protein from samples were separated via $12 \%$ sodium dodecyl sulfate-polyacrylamide gel electrophoresis (SDS-PAGE) at a voltage of $200 \mathrm{~V}$ and a current of $3 \mathrm{~A}$ and then transferred to a nitrocellulose membrane (Bio-Rad, Hercules, CA, USA) using the Trans-Blot ${ }^{\circledR}$ Turbo ${ }^{\mathrm{TM}}$ Transfer System (Bio-Rad, USA) at $15 \mathrm{~V}$ and $2.5 \mathrm{~A}$. Following transfer, the nitrocellulose membrane was blocked with $5 \%$ skimmed milk for 1 $\mathrm{h}$ at room temperature. The nitrocellulose membrane was then incubated with primary antibodies: anti- $\beta$ actin (1:1000, Cell signaling technologies 4970, USA), anti-p44/42 MAPK (Erk1 /2) (1:1000, Cell signaling technologies 4696, USA), anti-phospho- p44/42 MAPK (Erk1 /2) Primary antibody (1:1000, Cell signaling technologies 4370, USA), anti-Akt (1:1000), Cell signaling technologies 9272, USA), and anti-phosphoAkt (Thr308) (1:1000) (Cell signaling technologies 13038, USA). Membranes were incubated with primary antibody at $4^{\circ} \mathrm{C}$ on a shaker overnight. Subsequently, membranes were washed three times with TBS-Tween and incubated with horseradish-conjugated secondary anti-rabbit (1:1000, Cell signaling technology) or antimouse (1:1000, Cell signaling technology) secondary antibody for $1 \mathrm{~h}$. Membranes were washed three additional times with TBS-Tween. The chemiluminescent ECL reagent (Bio-Rad, USA) was added under darkness and was visualized utilizing the ChemiDoc Imaging system (Bio-Rad, USA).

\section{Statistical analysis}

All experiments were performed in triplicates and the results are expressed as the means \pm standard deviation of the triplicate assays utilizing Microsoft Excel and the 
Graphpad online calculator.

\section{RESULTS}

\section{Transfection efficiency}

The transfection efficiency of iMSC 3 cells with nontarget miRNA labelled with CY3 was assessed before the transfection process. The percentage of transfection was detected by the shift in signal indicated (Figs. 2A, 2B). The obtained transfection efficiency was $77.8 \%$, which indicated that miR-22-3p successfully transfected into iMSC3 cells under high efficiency.
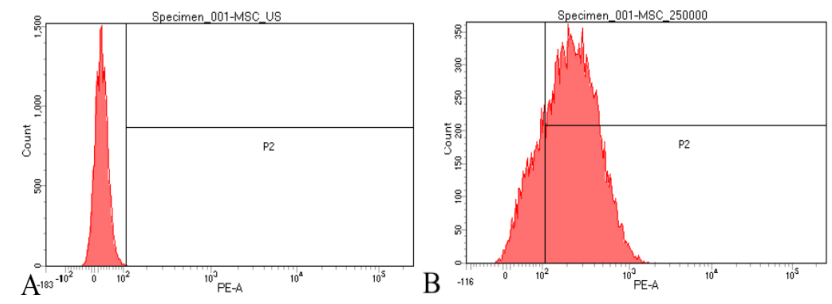

Fig. 2. iMSC3 cell transfection efficiency using flow cytometry. A, non-transfected iMSC3. B, iMSC3 cells transfected with non-target miRNA labeled with CY3.

miR-22-3p decreased adipocyte formation and lipid content in differentiated iMSC3

Morphological changes occur in iMSC3 cells following their differentiation into adipocytes. Lipid droplets start to form and can be visualized and compared between the control, negative control (transfected with non-target miRNA) and miR-22-3p transfected cell groups using fluorescent and inverted light microscopy. Oil red and Nile red stains were used to specifically stain fat or lipid droplets formed in adipocytes. DAPI was used to stain the cell nucleus. The lipid droplets from the control group are indicated by arrows in Figure 3A $(1,2,3,4)$, forming in higher amounts and larger in size in comparison to miR$22-3 p$ transfected cells shown in Figure 3A $(5,6,7,8)$. Merged DAPI and Nile red stains clearly indicated that lipid droplets formed around the nucleus. The negative control group also demonstrated higher lipid formation in comparison to miR-22-3p transfected cells. Furthermore, lipid contents (Fig. 3B) were measured from eluted oil red stains using a spectrophotometer. We found significant decreases $(p<0.05)$ in the amount of lipid in miR-22-3p transfected cells in comparison to control and negative control groups. Lipid counting was also performed on oil red stained fat droplets (Fig. 3C), where average fat droplet number $(\%)$ was significantly $(p<0.01, p<0.001)$ decreased in miR-22-3p transfected cells in comparison to control and negative control groups.

iMSC3 cells transfected with miR-22-3p upregulates nontargeted MAPK signaling during its differentiation into adipocytes

In this study, the effect of miR-22-3p transfection of iMSC3 cells into adipocytes was studied at the protein level. Several signaling pathways are involved in the differentiation process. The role of miR-22-3p in the MAPK and PI3-K signaling pathways was investigated at two time points during differentiation. The MAPK pathway is activated by dual phosphorylation of its residues (Thr 202/ Tyr 204). This activation interacts with downstream effector proteins that are involved in downstream cellular processing. We studied MAPK regulation after the first (4 days) (Fig. 4A, B and Fig. 1) and third differentiation cycles (12 days), respectively. (Fig. 4C, D and Fig. 1). Interestingly, we found that $\mathrm{p}-\mathrm{MAPK} / \mathrm{MAPK}$ expression levels were significantly upregulated $(p<0.05)$ at the above time intervals.

miR-22-3p did not regulate the PI3k/AKT signaling pathway during iMSC3 differentiation into adipocyte

The role of miR-22-3p in the PI3-K/Akt signaling pathway was investigated at two time points during differentiation. We studied PI3-K/Akt regulation after the first (4 days) (Fig. 5A, B and Fig. 1) and third differentiation cycles (12 days), respectively. (Fig. 5C, D and Fig. 1). We found that $\mathrm{p}-\mathrm{Akt}(\mathrm{Thr}) / \mathrm{Akt}$ expression levels were neither down- nor upregulated at the discussed time intervals. The obtained results were not statistically significant.

\section{DISCUSSION}

Obesity is a disorder characterized by an increase in adipose tissue fat mass (Wang et al., 2014). The process of adipogenesis involves differentiation of MSCs into adipocytes. The MSCs can be differentiated into in vitro adipocytes as well as an in vitro model of obesity. In our study, we found that transfection of miR-22-3p significantly suppressed the differentiation of iMSC3 cells into adipocytes. The iMSC 3 cells were transfected with non-target miRNA tagged with fluorescence (CY3) to assess transfection efficiency. The transfection rate was high, indicating cells could be easily transfected with microRNAs using RNAiMAX lipofectamine. A previous study reported the adipocyte inhibitory effects of miR-22 on adipose derived mesenchymal stem cells (hADMSC) (Huang et al., 2012). However, literature is lacking on the effect of miR-22-3p on the differentiation of iMSC3 into adipocytes. 
Oil Red
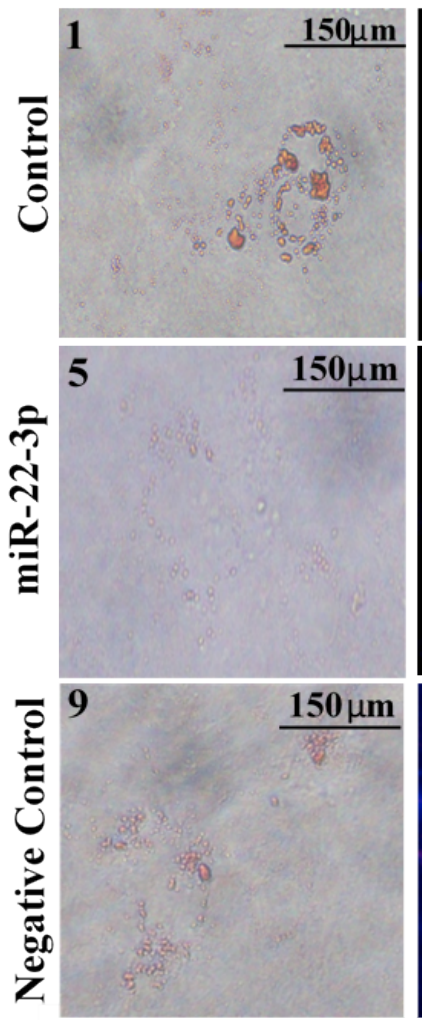

A

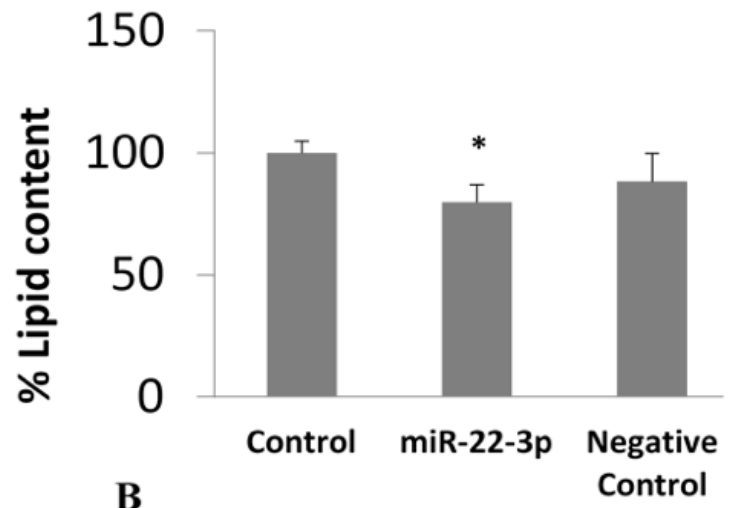

DAPI

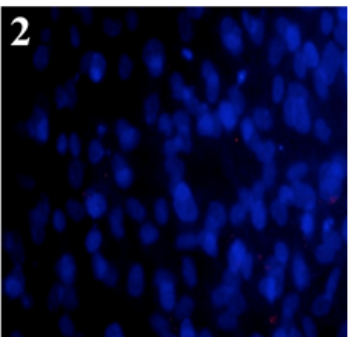

6
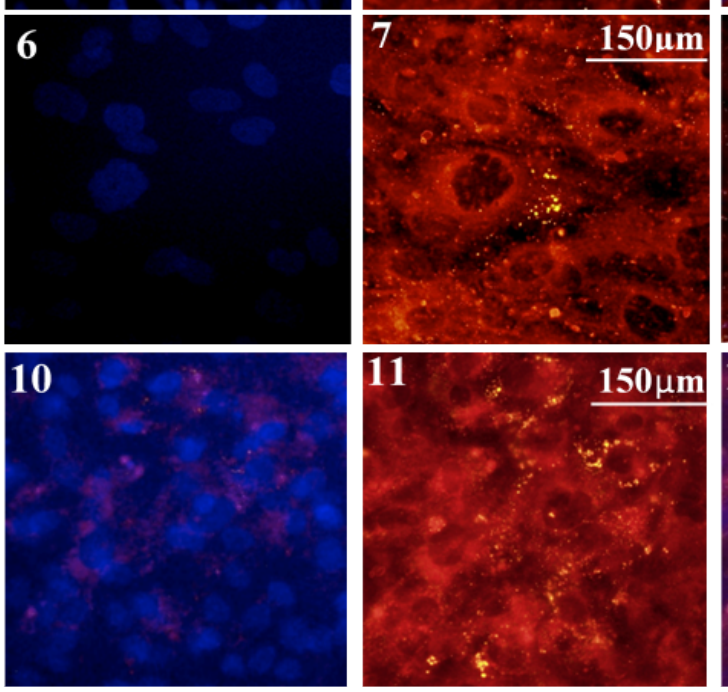
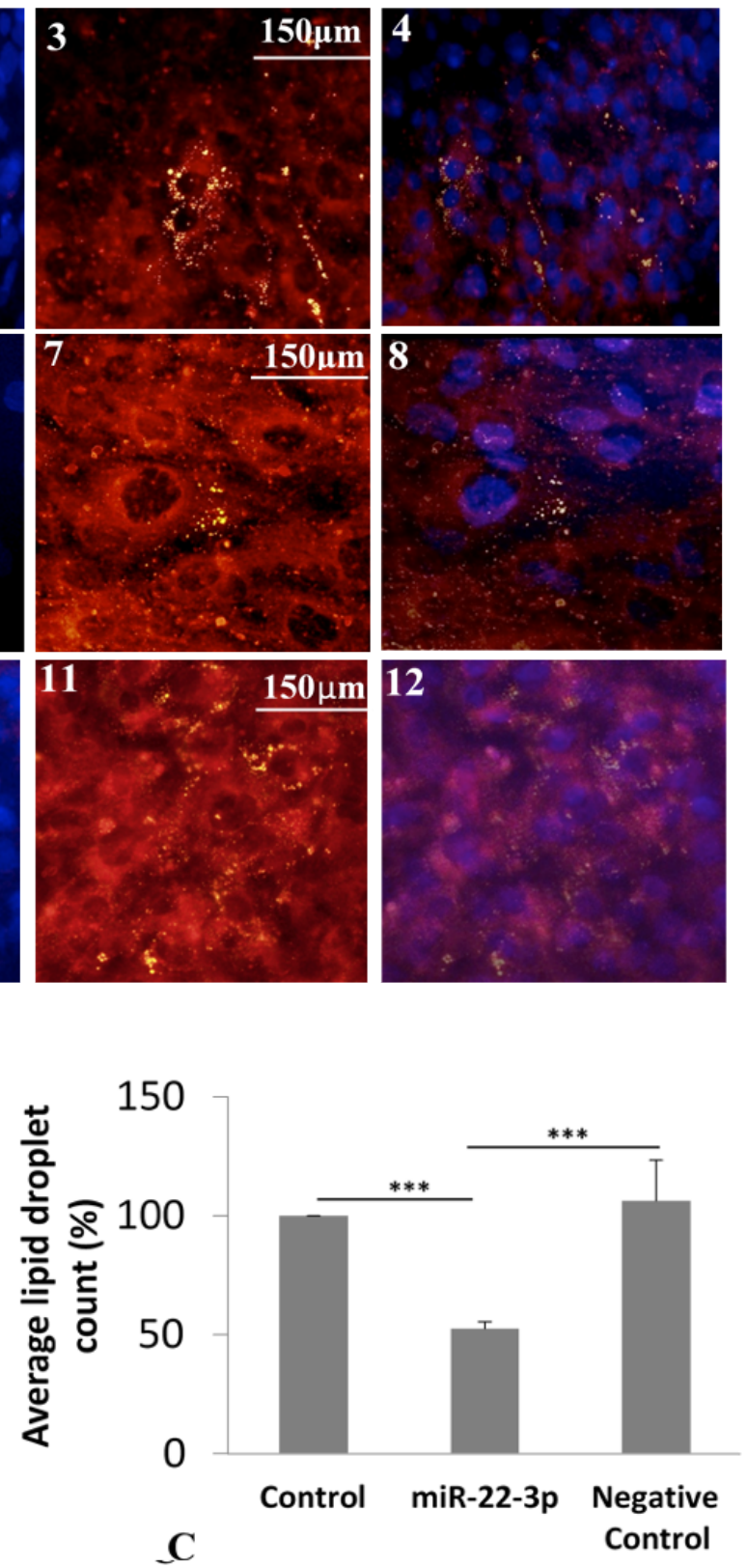

Fig. 3. Morphological effects of miR-22-3p transfection (lipid content and lipid droplet count) on the differentiation of iMSC3 cells into adipocyte. To determine the effect of the transfection into adipocyte, Oil red, DAPI and Nile red staining were performed. A, 1-4 shows control lipid droplets in oil red and Nile red, cell nucleus in DAPI, and merged DAPI/Nile red. 5-8, miR-22$3 p$ transfected cells and 9-11 negative control cells, respectively. B shows lipid content from iMSC3 cells differentiated into adipocytes. $\mathrm{C}$ shows the average number of fat droplets from different treatment groups. The experiments were performed in triplicates and the average percentage values were calculated. The data is presented as mean \pm S.D. $p=0.34,{ }^{*} \mathrm{p}<0.05,{ }^{* *} \mathrm{p}<0.01$, $* * * \mathrm{p}<0.001$ versus control or negative control groups. 

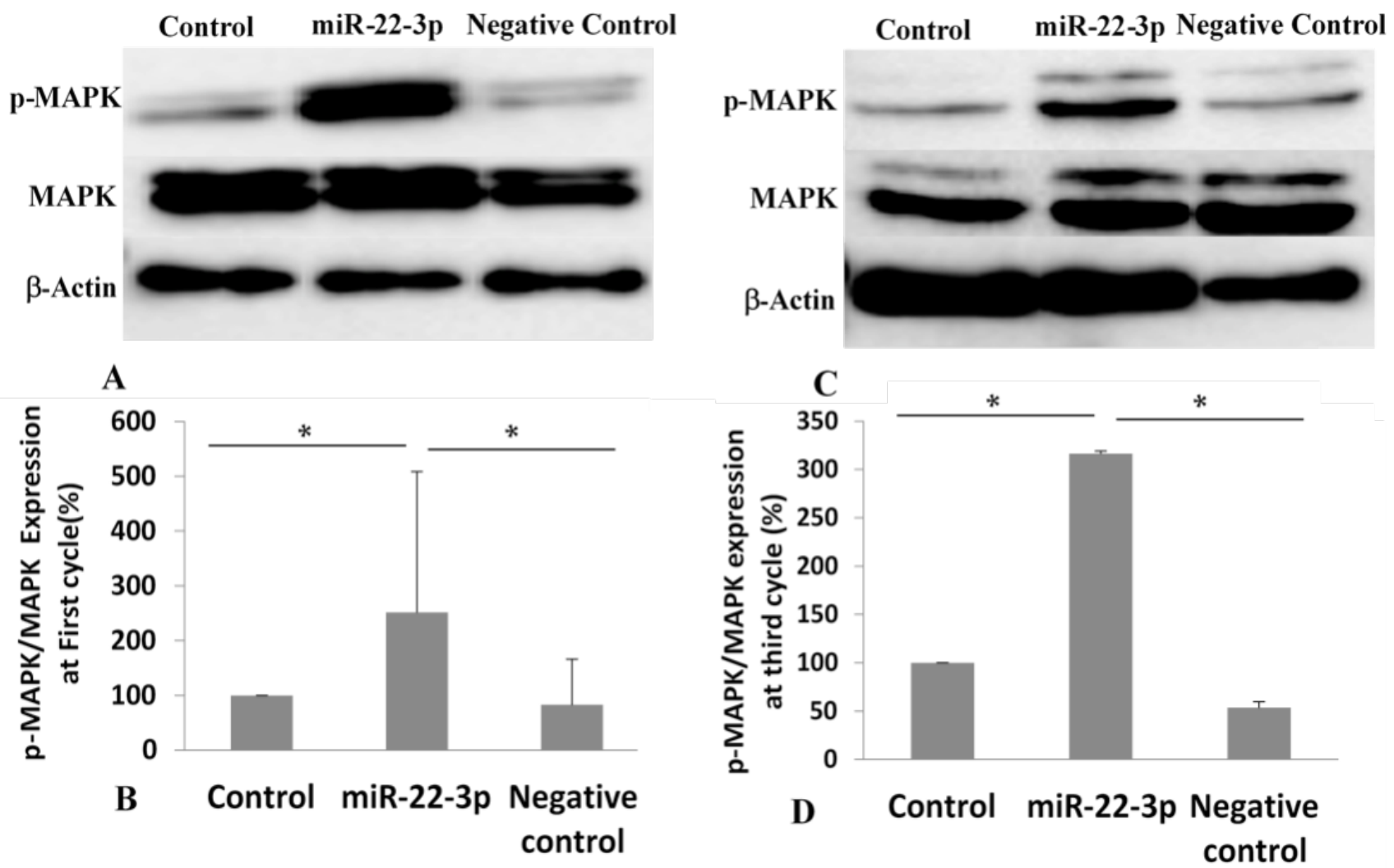

Fig. 4. Representative immunoblots showing expression of $\beta$-actin, total MAPK and phosphorylated MAPK along with graph of p-MAPK/MAPK relative expression. A, B, In the first cycle of differentiation to adipocytes, the immunoblots show an upregulation of p-MAPK in miR-22-3p transfected cells in comparison to control and negative control groups. C, D, Representative immunoblots and graph following the third cycle of differentiation. The results also indicate an upregulation of p-MAPK at this stage. Experiments were performed in triplicates and the average percentage values were calculated. The data is presented as mean \pm S.D $* \mathrm{p}<0.05$, versus control and negative control groups.

The transfection of miR-22-3p at the beginning of the process suppressed adipogenic differentiation as evident by the observed lower lipid content levels and low quantity of lipids in comparison to control and negative control groups. The lipid content assay confirmed that the transfection of miR-22-3p suppressed adipogenesis.

Target prediction indicates that more than 500 genes are targeted by miR-22-3p (Target Scan, 2019), including AKT3, a component of the PI3-K pathway. The PI3-K and MAPK signaling pathways are involved in multiple physiological processes, including; cell proliferation, differentiation, metabolism, cytoskeleton reorganization, cell death and survival (Pearson et al., 2001; Vivanco and Sawyers, 2002). AKT and phospho-AKT (Thr 308) western blots revealed an insignificant $p$-value at the end of the first and third cycles of differentiation, respectively. Hence, despite being a target of miR-22-3p, the transfection process did not affect the regulation of the PI3-K/AKT. Similarly, the transfection of miR-22-3p into cancer cells also led to non-regulation of PI3-K (Lv et al., 2018). Our study also found the analogous effects of miR-22-3p on the PI3-K/AKT pathway. Western blots confirmed that there was neither consistent up- nor downregulation of the pathway during differentiation of iMSC3 into adipocytes following miR-22-3p transfection.

Although the MAPK/ERK pathway is not a known target of miR-22-3p, our previous study found that this pathway enhances adipogenesis of iMSC3 under rosiglitazone treatment (Fayyad et al., 2019). Furthermore, PI3-K has been shown to interact with the MAPK pathway (Aksamitiene et al., 2012). A previous study found that ERK $1 / 2$ is involved in early stages of adipocyte differentiation (Marquez et al., 2017). It was our initial hypothesis that MAPK/ERK would be downregulated following miR-22-3p transfection as the latter suppresses adipocytes. However, the present study indicated that MAPK was paradoxically upregulated after transfection. Any signaling pathway 


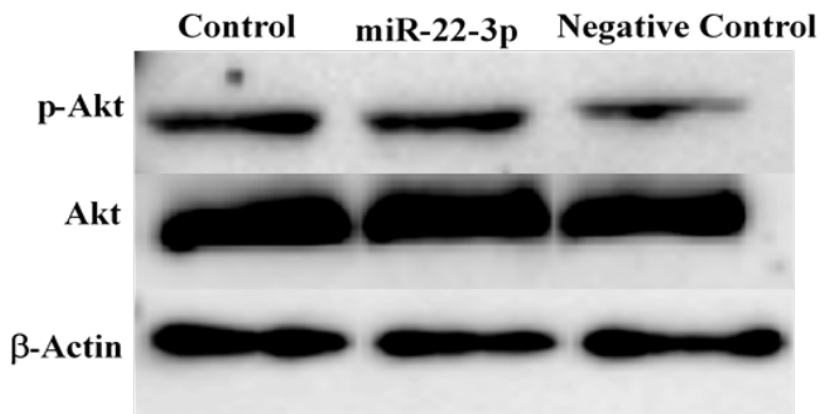

A

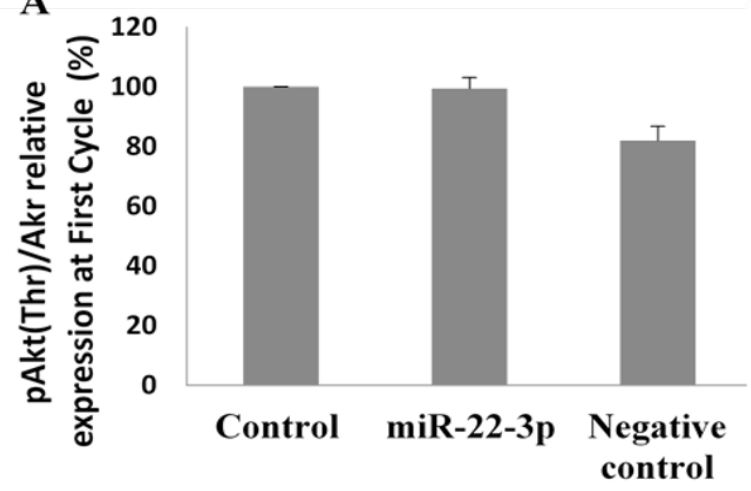

B

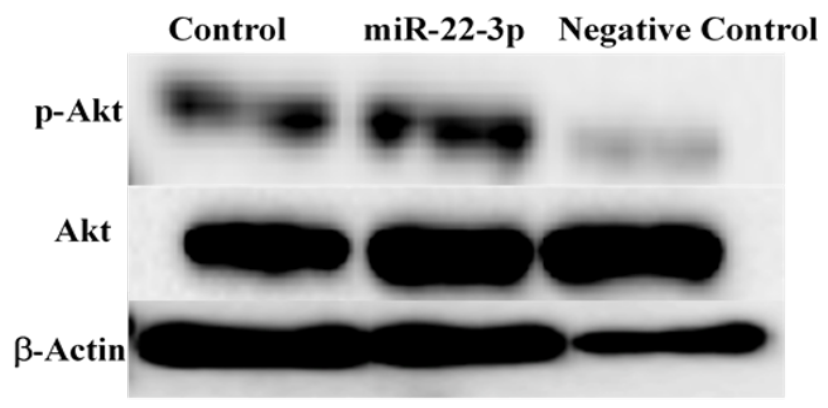

C

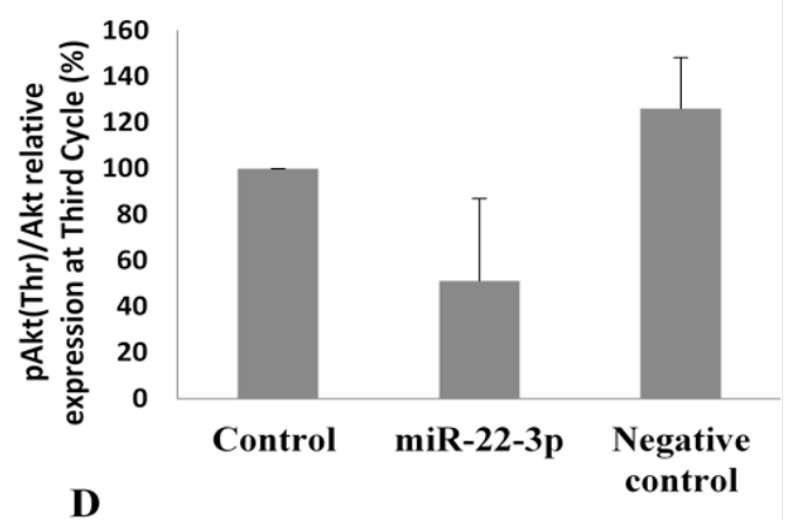

Fig. 5. Representative immunoblots showing expression of $\beta$-actin, total Akt and phosphorylated Akt (Thr) along with graphic representation of p-Akt/Akt relative expression (A, B). In the first cycle of differentiation to adipocytes, immunoblots show an upregulation of p-Akt in miR-22-3p transfected cells in comparison to control and negative control groups (C, D). Representative immunoblots and graph following the third cycle of differentiation also indicate an upregulation of p-Akt (Thr) at this stage. Experiments were performed in triplicates and the average percentage values were calculated. The data is presented as mean \pm S.D. The results were not statistically significant.

has diverse and often opposing effects in different cells (Lodish et al., 2000). Signaling pathways interact with effector proteins that have various effects depending on the context of the involved cells. As such, the regulation of more than 500 genes by miR-22-3p may also upregulate the MAPK/ERK pathway without having a direct effect on adipogenic differentiation.

\section{CONCLUSIONS}

Our study showed that miR-22-3p may have antiadipogenic effects on various cells. The transfection of miR-22-3p into iMSC3 cells suppressed adipogenesis without affecting the AKT3 protein, indicating no involvement of the PI-3/Akt pathway. At the same time, the MAPK pathway was observed to be indirectly upregulated following miR-22-3p transfection.

\section{Statement of conflicts of interest}

The authors have declared no conflict of interest.

\section{REFERENCES}

Aguena, M., Fanganiello, R.D., Tissiani, L.A.L., Ishiy, F.A.A., Atique, R., Alonso, N. and Passos-Bueno, M.R., 2012. Optimization of parameters for a more efficient use of adipose-derived stem cells in regenerative medicine therapies. Stem Cells Int., 2012. https://doi.org/10.1155/2012/303610

Aksamitiene, E., Kiyatkin, A. and Kholodenko, B.N., 2012. Cross-talk between mitogenic Ras/MAPK and survival PI3K/Akt pathways: A fine balance. Biochem. Soc. Trans., 40: 139-146. https://doi. org/10.1042/BST20110609

Castellano, E. and Downward, J., 2011. Ras interaction with PI3K: More than just another effector pathway. Genes Cancer, 2: 261-274. https://doi. org/10.1177/1947601911408079

Fayyad, A.M., Khan, A.A., Abdallah, S.H., Alomran, S.S., Bajou, K. and Khattak, M.N.K., 2019. Rosiglitazone enhances browning adipocytes in 
association with MAPK and PI3-K pathways during the differentiation of telomerase-transformed mesenchymal stromal cells into adipocytes. Int. J. mol. Sci., 20: e1618. https://doi.org/10.3390/ ijms20071618

Garg, S.K., Maurer, H., Reed, K. and Selagamsetty, R., 2014. Diabetes and cancer: Two diseases with obesity as a common risk factor. Diabetes Obes. Metab., 16: 97-110. https://doi.org/10.1111/ dom. 12124

Greenspan, P., Mayer, E.P. and Fowler, S.D., 1985. Nile red: A selective fluorescent stain for intracellular lipid droplets. J. Cell Biol., 100: 965-973. https:// doi.org/10.1083/jcb.100.3.965

Gregoire, F., Cynthia, M., Smas, M. and Sul, H.S., 1998. Understanding adipocyte differentiation. Physiol. Rev., 78: 783-809. https://doi.org/10.1152/ physrev.1998.78.3.783

Han, J., Choi, H.Y., Dayem, A.A., Kim, K., Yang, G., Won, J., Do, S.H., Kim, J.H., Jeong, K.S. and Cho, S.G., 2017. Regulation of adipogenesis through differential modulation of ROS and kinase signaling pathways by 3,4'-dihydroxyflavone treatment. J. cell. Biochem., 118: 1065-1077. https://doi.org/10.1002/jcb.25681

Hartig, S.M., Hamilton M.P., Bader D.A. and McGuire, S.E., 2015. The microRNA interactome in metabolic homeostasis. Trends Endocrinol. Metab., 26: 733 745. https://doi.org/10.1016/j.tem.2015.09.006

Huang, S., Wang, S., Bian, C., Yang, Z., Zhou, H., Zeng, Y., Li, H., Han, Q. and Zhao, R.C., 2012. Upregulation of miR-22 promotes osteogenic differentiation and inhibits adipogenic differentiation of human adipose tissue-derived mesenchymal stem cells by repressing HDAC6 protein expression. Stem Cells Dev., 21: 25312540. https://doi.org/10.1089/scd.2012.0014

Huat, T.J., Khan, A.A., Abdullah, J.M., Idris, F.M. and Jaafar, H., 2015. MicroRNA expression profile of neural progenitor-like cells derived from rat bone marrow mesenchymal stem cells under the influence of IGF-1, bFGF and EGF. Int. J. mol. Sci., 16: 9693-9718. https://doi.org/10.3390/ ijms 16059693

Lodish, H., Berk, A., Zipursky, S.L., Matsudaira, P., Baltimore, D. and Darnell, J., 2000. Molecular cell biology. $4^{\text {th }}$ edition. W.H. Freeman \& Co Ltd New York

Luo, L.J., Zhang, L.P., Duan, C.Y., Wang, B., He, N.N., Abulimiti, P. and Lin, Y., 2017. The inhibition role of miR-22 in hepatocellular carcinoma cell migration and invasion via targeting CD147.
Cancer Cell Int., 17: 1-9. https://doi.org/10.1186/ s12935-016-0380-8

Lv, K.T., Liu, Z., Feng, J., Zhao, W., Hao, T., Ding, W.Y., Chu, J.P. and Gao, L.J., 2018. MiR-22$3 \mathrm{p}$ regulates cell proliferation and inhibits cell apoptosis through targeting the eif4EBP3 gene in human cervical squamous carcinoma cells. Int. J. med. Sci., 15: 142-152. https://doi.org/10.7150/ ijms. 21645

Marquez, M.P., Alencastro, F., Madrigal, A., Jimenez, J.L., Blanco, G., Gureghian, A., Keagy, L., Lee, C., Liu, R., Tan, L. and Deignan, K., 2017. The role of cellular proliferation in adipogenic differentiation of human adipose tissue-derived mesenchymal stem cells. Stem Cells Dev., 26: 1578-1595. https:// doi.org/10.1089/scd.2017.0071

Noriyoki, O., Parker, J.L., Lugus, J.J. and Walsh, K., 2011. Adipokines in inflammation and metabolic disease. Nat. Rev. Immunol., 11: 85-97. https://doi. org/10.1038/nri2921

Ortega, F.J., Mercader, J.M., Catalán, V., MorenoNavarrete, J.M., Pueyo, N., Sabater, M., GómezAmbrosi, J., Anglada, R., Fernández-Formoso, J.A., Ricart, W. and Frühbeck, G., 2013. Targeting the circulating microRNA signature of obesity. Clin. Chem., 59: 781-792. https://doi.org/10.1373/ clinchem.2012.195776

Pearson, G., Robinson, F., Gibson, T.B., Xu, B.E., Karandikar, M., Berman, K. and Cobb, M.H., 2001. Mitogen-activated protein (MAP) kinase pathways: Regulation and physiological functions. Endocr. Rev., 22: 153-183. https://doi.org/10.1210/ edrv.22.2.0428

Ruiz-Ojeda, F.J., Rupérez, A.I., Gomez-Llorente, C., Gil, A. and Aguilera, C.M., 2016. Cell models and their application for studying adipogenic differentiation in relation to obesity: A review. Int. J. mol. Sci., 17: 1-26. https://doi.org/10.3390/ ijms 17071040

Sousa-nunes, R. and Somers, W.G., 2013. Mechanisms of asymmetric progenitor divisions. Transcr. Transl. Regul. Stem Cells, 786: 329-351. https:// doi.org/10.1007/978-94-007-6621-1_6

Target Scan. 2019. Accessed October 1. www. targetscan.com.

Vivanco, I. and Sawyers, C.L., 2002. The phosphatidylinositol 3-kinase-AKT pathway in humancancer. Nat. Rev. Cancer, 2: 489-501. https://doi.org/10.1038/nrc839

Wang, Q.A., Scherer, P.E. and Gupta, R.K., 2014. Improved methodologies for the study of adipose biology: Insights gained and opportunities ahead. 
J. Lipid Res., 55: 605-624. https://doi.org/10.1194/ jlr.R046441

Xiong, J., Yu, D., Wei, N., Fu, H., Cai, T., Huang, Y., Wu, C., Zheng, X., Du, Q., Lin, D. and Liang, Z., 2010. An estrogen receptor $\alpha$ suppressor, microRNA-22, is downregulated in estrogen receptor $\alpha$-positive human breast cancer cell lines and clinical samples. FEBS J., 277: 1684-1694. https://doi.org/10.1111/ j.1742-4658.2010.07594.x

Yang, J., Fan, Z., Yang, J., Ding, J., Yang, C. and Chen, L., 2016. MicroRNA-22 attenuates myocardial ischemia-reperfusion injury via an anti-inflammatory mechanism in rats. Exp. Ther.
Med., 3t3 12: 3249-3255. https://doi.org/10.3892/ etm.2016.3777

Zebisch, K., Voigt, V., Wabitsch, M. and Brandsch, M., 2012. Protocol for effective differentiation of 3T3L1 cells to adipocytes. Anal. Biochem. Elsevier. Inc., 425: 88-90. https://doi.org/10.1016/j. ab.2012.03.005

Zhenhui, C., 2012. Growth arrest induction of 3T3L1 preadipocytes by serum starvation and their differentiation by the hormonal adipogenic cocktail. J. Cell Anim. Biol., 6: 57-65. https://doi. org/10.5897/JCAB11.074 